\section{Case Reports in Ophthalmology}

\title{
Adult Coats Disease Presenting as Subfoveal Nodule
}

\author{
Salim Z. Asaad ${ }^{a} \quad$ Nazimul Hussain ${ }^{b}$ \\ aMagrabi Eye and Ear Center, Abu Dhabi, United Arab Emirates; ${ }^{b}$ Department of \\ Ophthalmology, Al Zahra Hospital, Sharjah, United Arab Emirates
}

\section{Keywords}

Coats disease $\cdot$ Subfoveal nodule $\cdot$ Macular fibrosis - Telangiectasia $\cdot$ Exudation

\begin{abstract}
A 21-year-old female presented with decreased vision in the right eye. Best corrected visual acuity was $6 / 60$ in the right eye and 6/6 in the left eye. Funduscopy of the right eye revealed a subfoveal nodule with surrounding exudates and temporal peripheral retinal vessel telangiectasia with exudation. The patient underwent retinal laser in the areas of telangiectatic vessels and capillary non-perfusion as seen on fundus fluorescein angiography. It was combined with an intravitreal injection of bevacizumab which was repeated twice at monthly intervals. The macular lesion and peripheral vessels along with subretinal exudates showed resolution during the course of treatment. However, the patient was lost to follow-up and returned 5 months later when examination revealed increased macular fibrosis. In the literature, the reported presentation of subfoveal nodule in Coats disease is during the first decade. It progresses to macular fibrosis within a few months. This case illustrates that subfoveal nodule before macular fibrosis in Coats disease may present later, even up to the third decade.
\end{abstract}




\section{Introduction}

Coats disease is a sporadic condition characterized by idiopathic retinal vessel telangiectasia and exudation, usually occurring unilaterally in young males [1]. Often there is macular exudation which when severe may sometimes present as a fibroglial nodule [2]. The term "subfoveal nodule" has been used to describe such a lesion, which is usually associated with a vascular component within the fovea or the posterior pole of patients with Coats disease $[3,4]$. In the literature, the reported presentation of subfoveal nodule in Coats disease is during the first decade [3-6]. We report a 21-year-old adult with Coats disease who presented with a subfoveal nodule.

\section{Case Report}

A 21-year-old female presented to us with decreased vision in the right eye that was revealed during a recent eye examination. Visual acuity recorded was $6 / 60$ in the right eye and $6 / 6$ in the left eye. On examination, ocular alignment showed 30-degree exotropia in the right eye; ocular movements were full in all direction of gaze. Anterior segment examination was unremarkable in both eyes. Fundus examination of the right eye showed a yellowishwhite raised spheroidal lesion approximately 1 disc in diameter at the macula with surrounding subretinal exudates (Fig. 1a). Retinal arteries and veins dipping into the lesion were observed. The temporal and infero-temporal periphery showed retinal vessel telangiectasia with subretinal exudation. Fundus examination of the left eye was unremarkable. Fundus fluorescein angiography showed hypofluorescence of the subfoveal lesion in early and mid-phase which became hyperfluorescent with minimal leakage in late phase (Fig. $1 \mathrm{~b}, \mathrm{c})$. Retinal-retinal anastomosis was demonstrated within the nodule. Peripheral images showed telangiectatic vessels, capillary nonperfusion, and leakage. Optical coherence tomography showed a hyperreflective subfoveal lesion with posterior shadowing and elevation of the overlying retinal layers (Fig. 1d). The height of the lesion was approximately 1,000 $\mu \mathrm{m}$.

The patient underwent laser photocoagulation in the peripheral retinal areas of capillary nonperfusion and telangiectatic vessels. Additionally, an intravitreal injection of $1.25 \mathrm{mg}$ of bevacizumab was given. She underwent additional laser treatment elsewhere before following up with us 4 weeks later, when examination showed laser scars surrounding the lesion at the macula. The lesion appeared less vascular and the exudates seemed to have decreased (Fig. 2a). The peripheral vessels and subretinal exudation also showed regression (Fig. 2b). However, there was no evident visual improvement. A second dose of $1.25 \mathrm{mg}$ of Intravitreal Bevacizumab was given. Follow-up examination at 8 weeks showed a further decrease in vascularity and subretinal exudates and a reduction in the size of the macular lesion (Fig. 2c). Thereafter, the patient was lost to follow-up and only returned 5 months later when examination showed that the macular lesion appeared more fibrotic (Fig. 2d) with increased subretinal exudates. She declined any further intervention.

\section{Discussion}

In Coats disease there is often accumulation of exudation in the macular region. Dense macular exudation can present as a nodular fibrotic lesion commonly referred to as a "subfoveal nodule" [4-6] which, as mentioned by Shields et al. [2], augurs a poor visual progno- 
sis. Subfoveal nodule at the macula invariably progresses to macular fibrosis and scarring within a few months $[3,7,8]$. Daruich et al. [3] reviewed 21 eyes with stage $2 \mathrm{~B}$ or $3 \mathrm{~A} 1$ Coats disease and found the mean time from the diagnosis of subfoveal nodule to the detection of macular fibrosis was $11.0 \pm 2.6$ months. They reported the mean age at presentation of subfoveal nodule was $4.9 \pm 3.5$ years. Presentation of subfoveal nodule in the paediatric age group has also been reported by other authors [4-6]. In our case a subfoveal nodule was observed in a 21-year-old adult female with Coats disease. The subfoveal nodule had not progressed to macular fibrosis at the time of presentation. This case represents a more delayed presentation of subfoveal nodule but before the onset of macular fibrosis in Coats disease.

Subfoveal nodules are usually associated with a vascular component within the posterior pole as was also observed in our case. Daruich et al. [3] observed a second-to-third-order artery and vein entering the subfoveal nodule in all of the 21 cases they reviewed. Observation of vascular anastomosis at the macula adds credence to the concept that the disease is part of a clinical spectrum (of primary retinal telangiectasias) which also includes idiopathic macular telangiectasia [9]. Sigler and Calzada [5] reported the presence of retinal angiomatous proliferation with chorioretinal anastomosis using spectral domain optical coherence tomography in 5 of $21(24 \%)$ patients with Coats disease treated by intravitreal injection of bevacizumab. The authors hypothesized that Coats disease involves an abortive defect during the angiogenesis of the retinal midcapillary plexus, and that this aberrant vascular development in the macula could lead to type 3 neovascularization with chorioretinal anastomosis. Jumper et al. [7] described the development of macular fibrosis in 11 of 47 patients $(23 \%)$ with Coats disease and its association with intraretinal anastomosis and neovascularization. These authors proposed that macular fibrosis resulted from a neovascular process in response to the accumulation of lipid exudates.

Subfoveal nodules probably form due to severe exudation and accumulation at the macula. They have never been observed independent of peripheral telangiectasia. Exudation from the macular telangiectasia may contribute to their formation, and therefore treatment by intravitreal anti-vascular endothelial growth factor (anti-VEGF) agents may be a relevant strategy. Anti-VEGFs have been used as an effective adjunct in the treatment of Coats disease for the management of exudate, macular oedema, and serous retinal detachment [10].

\section{Conclusion}

The reported presentation of subfoveal nodule in Coats disease is during the first decade. It progresses to macular fibrosis within a few months. This case illustrates that subfoveal nodule before the onset of macular fibrosis in Coats disease may present at a later age, even up to the third decade.

\section{Statement of Ethics}

Institutional Ethics Committee permission was granted for the preparation and publication of this paper. 


\section{Disclosure Statement}

The authors report no conflict of interest in relation to this work.

\section{References}

1 Coats G: Forms of retinal diseases with massive exudation. R Lond Ophthalmol Hosp Rep 1908;17:440525.

2 Shields JA, Shields CL, Honavar SG, et al: Classification and management of Coats disease: the 2,000 proctor lecture. Am J Ophthalmol 2001;131:572-583.

3 Daruich AL, Moulin AL, Tran HV, Matet A, Munier FL: Subfoveal nodule in Coats' disease: toward an updated classification predicting visual prognosis. Retina 2017;37:1591-1598.

4 Khurana RN, Samuel MA, Murphree AL, et al: Subfoveal nodule in Coats' disease. Clin Exp Ophthalmol 2005;33:301-302.

5 Sigler EJ, Calzada JI: Retinal angiomatous proliferation with chorioretinal anastomosis in childhood Coats disease: a reappraisal of macular fibrosis using multimodal imaging. Retina 2015;35:537-546.

6 Chang MM, McLean IW, Merritt JC: Coats' disease: a study of 62 histologically confirmed cases. J Pediatr Ophthalmol Strabismus 1984;21:163-168.

7 Jumper JM, Pomerleau D, McDonald HR, et al: Macular fibrosis in Coats disease. Retina 2010;30:S9-S14.

8 Vezzola D, Mapelli C, Canton V, Viola F, Ratiglia R: Macular fibrosis in Coats disease. Retina 2011;31:2136-2137.

9 Perez MA, Shechtman DL, Gurwood A: The continuum of primary retinal telangiectasia. Optometry 2011;82:15865.

10 Sigler EJ, Randolph JC, Calzada JI, et al: Current management of Coats disease. Surv Ophthalmol 2014;59:30-46. 


\section{Case Reports in Ophthalmology}
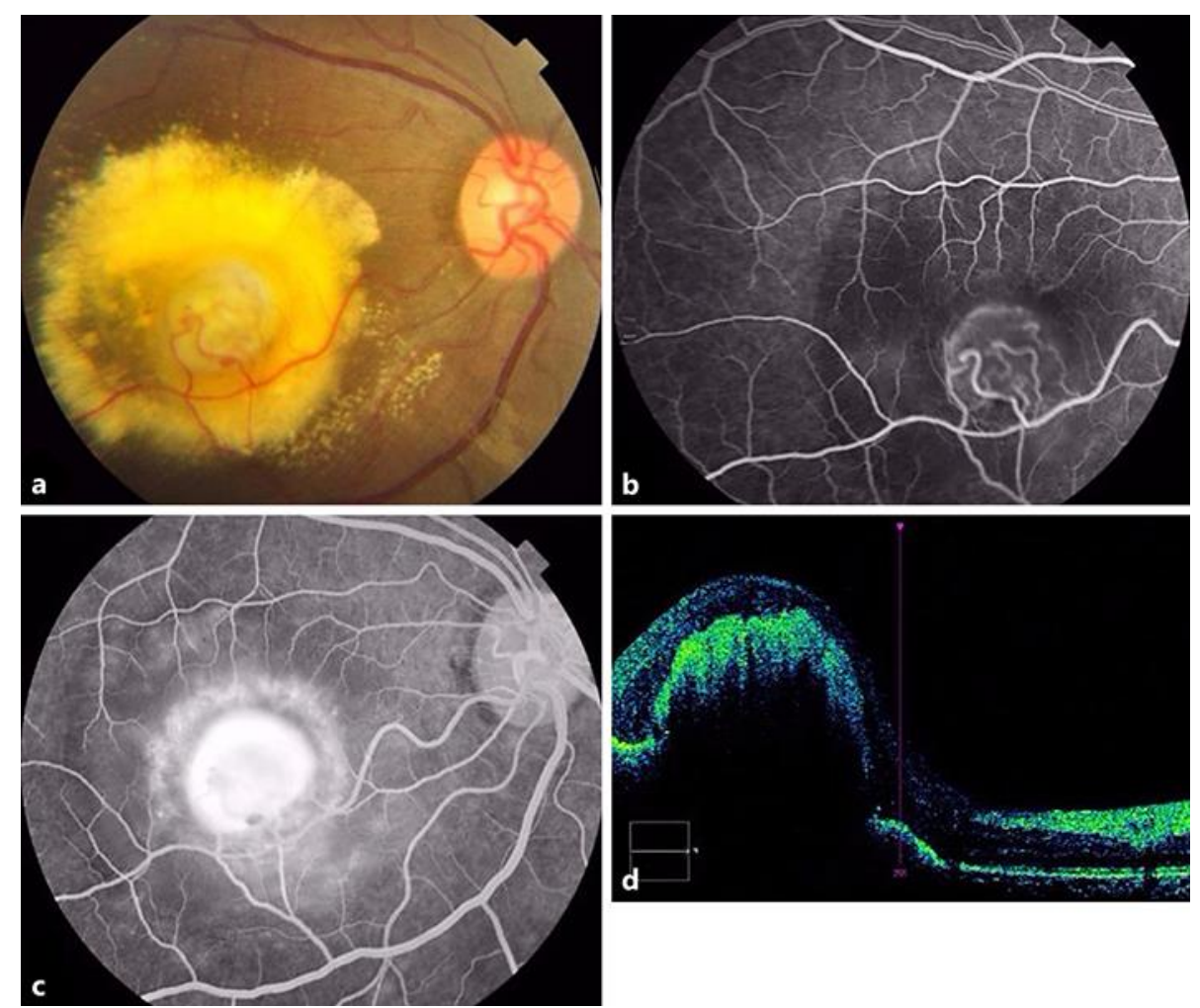

Fig. 1. a Colour fundus photograph showing a yellowish-white raised spheroidal lesion at the macula with surrounding subretinal exudates. b c Fundus fluorescein angiogram showing hypofluorescence of the subfoveal lesion in early and mid-phase which became hyperfluorescent with minimal leakage in late phase. d Optical coherence tomography showing a hyperreflective subfoveal lesion with posterior shadowing. 


\section{Case Reports in Ophthalmology}
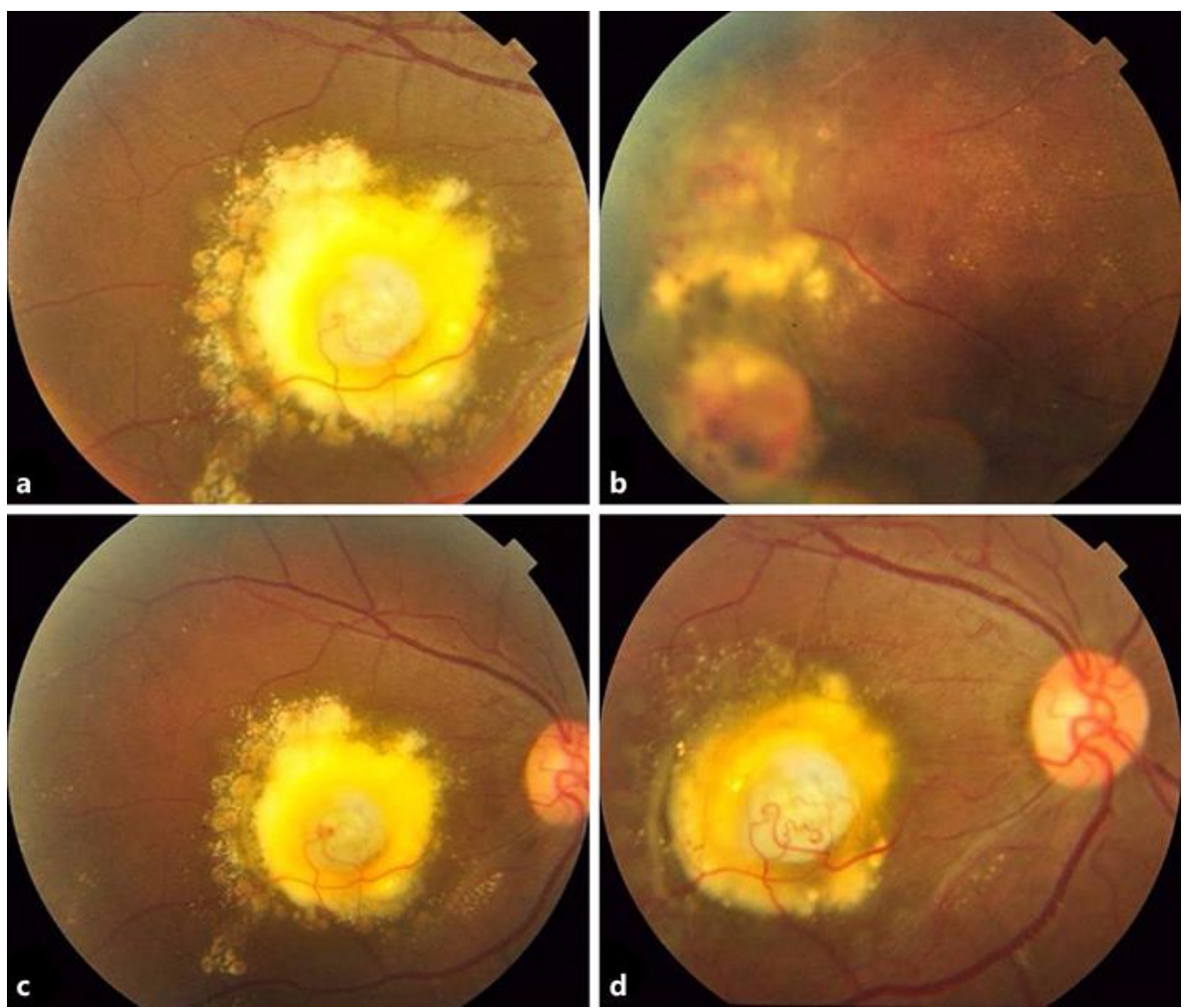

Fig. 2. Colour fundus photographs at 4-week follow-up showing resolution of the macular lesion and subretinal exudates with surrounding laser scars (laser treatment was done elsewhere) (a) and resolution of the peripheral vessels and subretinal exudation (b). c Follow-up at 8 weeks showing further resolution of the macular lesion and subretinal exudates. $\mathbf{d}$ Colour fundus photograph 5 months later showing macular fibrosis. 